\title{
Escalas para avaliação da sobrecarga de cuidadores de pacientes com Acidente Vascular Encefálico
}

\author{
Scales for evaluation of the overload of caregivers of patients with Stroke
}

Escalas para evaluación de la sobrecarga de cuidadores de pacientes con Accidente Vascular Encefálico

\section{Ana Railka de Souza Oliveira', Alice Gabrielle de Sousa Costa', Vanessa Emille Carvalho de Sousa", Thelma Leite de Araujo"', Viviane Martins da Silva"', Marcos Venícios de Oliveira Lopes"', Maria Vera Lúcia Moreira Leitão Cardoso"II}

\author{
' Universidade Federal do Ceará, Departamento de Enfermagem, Programa de Pós-Graduação em Enfermagem \\ (Doutoranda). Fortaleza-CE, Brasil. \\ "Instituto Florence de Ensino Superior, Curso de Graduação em Enfermagem. São Luís-MA, Brasil \\ I' Universidade Federal do Ceará, Departamento de Enfermagem, Programa de Pós-Graduação em Enfermagem. \\ Fortaleza-CE, Brasil.
}

\author{
Submissão: 20-04-2011 Aprovação: 22-10-2012
}

\section{RESUMO}

O objetivo do estudo foi avaliar as escalas disponíveis na literatura para medir a sobrecarga de cuidadores de pacientes acometidos por acidente vascular encefálico. Realizou-se uma revisão bibliográfica nas três bases de dados: LILACS, CINAHL e SCOPUS. Selecionaram-se 23 trabalhos e identificaram-se 24 diferentes escalas. Destas, as mais citadas foram o Caregiver Strain Index, a Caregiver Burden Scale, a Caregiver Reaction Assessment, o Sense of Competence Questionnaire, a Relatives Stress Scale a Zarit Burden Interview. O uso de escalas para mensurar a sobrecarga é uma ferramenta importante para avaliar o contexto no qual está inserido o cuidador, entretanto é mais fidedigno quando associado a outros instrumentos de mensuração. Portanto, é fundamental pesquisas de validação de escalas para essa população.

Descritores: Acidente Vascular Cerebral; Cuidadores; Tecnologia; Escala.

\section{ABSTRACT}

The objective of the study was to evaluate the available productions in literature about scales to measure the overload of caregivers of patients with stroke. It was carried out a bibliographical revision in three databases: LILACS, CINAHL and SCOPUS. In 23 works, 24 different scales were identified, and the most cited were: the Caregiver Strain Index, the Caregiver Burden Scale, the Caregiver Reaction Assessment, the Sense of Competence Questionnaire, the Relatives Stress Scale and the Zarit Burden Interview. The use of scales to measure the overload is an important tool to evaluate the context in which the caregiver is inserted, but is more trustworthy to use more than one measure instrument than use only one. Therefore, the validation of scales becomes important for this population.

Key words: Stroke; Caregivers; Technology; Scales.

\section{RESUMEN}

El objetivo de esto estudio fue evaluar las escalas disponibles en literatura para evaluar la sobrecarga de cuidadores de pacientes acometidos por accidente vascular encefálico. Una revisión bibliográfica fue desarrollada en tres bases de datos: LILAS, CINAHL y SCOPUS. En 23 trabajos seleccionados, 24 diferentes escalas fueron identificadas, siendo las principales: el Caregiver Strain Index, la Caregiver Burden Scale, el Caregiver Reaction Assessment, el Sense of Competence Questionnaire, la Relatives Stress Scale y la Zarit Burden Interview. El uso de escalas para mensurar sobrecarga es una herramienta importante en el contexto donde se inserta el cuidador. Es más confiable utilizar más que uno instrumento de mensuración. Por esto, la validación de escalas es importante para esta población.

Palabras clave: Accidente Cerebro Vascular; Cuidadores; Tecnología; Escala. 


\section{INTRODUÇÃO}

A avaliação da sobrecarga dos cuidadores familiares de pacientes acometidos por acidente vascular encefálico (AVE) tornou-se necessária em virtude do maior envolvimento das famílias nos cuidados cotidianos a esses pacientes.

Trabalhos de revisão de literatura apontaram que a sobrecarga dos familiares resulta das tarefas advindas do papel de cuidador e das mudanças ocorridas em sua vida social e profissional, pois eles priorizam as necessidades dos pacientes. Além disso, conforme esses estudos mostraram, a sobrecarga é agravada pela falta de informação a respeito da doença do paciente, do tratamento utilizado e das estratégias mais adequadas para lidar com os comportamentos problemáticos dos pacientes e para o manejo das situações de crise ${ }^{(1-2)}$.

A sobrecarga do cuidador pode ser vista como um conceito vasto que abrange a esfera biopsicossocial e resulta da busca de um equilíbrio entre estas variáveis: tempo disponível para o cuidado, recursos financeiros, condições psicológicas, físicas e sociais, atribuições e distribuição de papéis ${ }^{(2-3)}$. Logo, exigem-se instrumentos de medida amplos para identificar os domínios mais afetados.

Em face desta situação, os profissionais de enfermagem devem começar a desenvolver instrumentos de medidas que são tecnologias apropriadas e com possibilidades emancipatórias, procurando se informar e divulgar as experiências vividas em seus locais de trabalho, para transformar a utilização empírica em uma abordagem científica ${ }^{(4)}$.

Assim, a adoção de escalas como uma forma de tecnologia pode ser o resultado de processos concretizados a partir da experiência cotidiana e da pesquisa, para o desenvolvimento de um conjunto de conhecimento/saberes organizados, para o emprego no processo de concepção, elaboração, planejamento, execução/operacionalização e manutenção de serviços produzidos e controlados, com uma finalidade prática específica ${ }^{(4)}$.

Diante desse cenário, o objetivo do presente estudo foi localizar e avaliar as produções disponíveis na literatura sobre escalas para mensurar a sobrecarga de cuidadores de pacientes acometidos por acidente vascular encefálico.

\section{METODOLOGIA}

Com vistas à elaboração desta revisão narrativa da literatura percorreram-se as seguintes etapas: estabelecimento dos objetivos da revisão; estabelecimento de critérios de inclusão e exclusão de artigos; definição das informações a serem extraídas dos artigos selecionados; análise dos resultados; discussão e apresentação dos resultados e, por fim, a apresentação da revisão( ${ }^{(5)}$.

Para nortear a revisão, formulou-se esta questão: quais escalas têm sido utilizadas para avaliar a sobrecarga de cuidadores de pacientes acometidos por acidente vascular encefálico?

Para a seleção dos artigos usaram-se três bases de dados: Literatura Latino-Americana e do Caribe em Ciências da Saúde (LILACS), Cumulative Index to Nursing and Allied Health Literature (CINAHL) e Base de dados multidisciplinar (SCOPUS). O acesso às bases de dados ocorreu por meio do portal de periódicos da Coordenação de Aperfeiçoamento de Pessoal de Nível Superior (CAPES). Dessa forma, procurou-se ampliar o âmbito da pesquisa, minimizando possíveis vieses nessa etapa do processo de elaboração da revisão.

Os critérios de inclusão dos artigos definidos, inicialmente, para a presente revisão foram: artigos que mensurassem a sobrecarga de cuidadores de pacientes acometidos por AVE; artigos publicados em português, inglês e espanhol, com os resumos disponíveis nas bases de dados selecionadas e com texto na íntegra disponível eletronicamente e gratuitamente.

Apesar das características específicas para o acesso às bases de dados selecionadas, as estratégias adotadas para localizar os artigos foram semelhantes, tendo como eixo norteador a pergunta e os critérios de inclusão da revisão integrativa, previamente estabelecidos, para manter a coerência na busca dos artigos e evitar possíveis vieses. As palavras-chave utilizadas foram sobrecarga do cuidador e acidente vascular cerebral ou caregiver burden and stroke para o LILACS e caregiver burden and stroke para o CINAHL e a SCOPUS.

Na base de dados LILACS, encontrou-se um trabalho. Este, porém, não foi selecionado para o estudo por não utilizar escalas para mensurar a sobrecarga dos cuidadores de pacientes após AVE, pois se tratava de uma pesquisa qualitativa.

No SCOPUS, foram encontrados 73 trabalhos, dos quais

\begin{tabular}{|c|l|l|c|c|c|}
\hline $\begin{array}{c}\text { Base de } \\
\text { dados }\end{array}$ & Palavras chave & Método de busca & $\begin{array}{c}\text { Resultados } \\
\text { obtidos }\end{array}$ & $\begin{array}{c}\text { Quantidade de } \\
\text { artigos selecio- } \\
\text { nados }\end{array}$ & $\begin{array}{c}\text { Quantidade de } \\
\text { artigos avaliados }\end{array}$ \\
\hline CINAHL & caregiver burden and stroke & $\begin{array}{l}\text { Busca utilizando operador booleano } \\
\text { "and" entre as palavras }\end{array}$ & 73 & 21 & 41 \\
\hline \multirow{2}{*}{ SCOPUS } & caregiver burden and stroke & $\begin{array}{l}\text { Busca utilizando operador booleano } \\
\text { "and" entre as palavras }\end{array}$ & 41 & 0 \\
\hline \multirow{2}{*}{ LILACS } & caregiver burden and stroke & $\begin{array}{l}\text { Busca utilizando operador booleano } \\
\text { "and" entre as palavras }\end{array}$ & 1 & 0 & 0 \\
\cline { 2 - 6 } & $\begin{array}{l}\text { sobrecarga cuidador e acidente } \\
\text { vascular cerebral }\end{array}$ & $\begin{array}{l}\text { Busca utilizando operador booleano } \\
\text { "and" entre as palavras }\end{array}$ & 1 & 0 \\
\hline
\end{tabular}

Quadro 1 - Síntese dos trabalhos selecionados pela busca nas bases de dados científicas. Fortaleza, 2009. 
41 estavam na íntegra. Desses, foram selecionados e avaliados nove após a leitura do título e dos resumos disponíveis.

A consulta na base de dados do CINAHL forneceu 253 trabalhos. Apenas 73 possuíam o trabalho na integra e 21 foram selecionados após a leitura do título e dos resumos disponíveis. Contudo, quatro foram excluídos por estarem repetidos e três por não avaliar a sobrecarga. Restaram, pois, 14 artigos para avaliação. No Quadro 1, será apresentada uma síntese da busca nas bases de dados.

Para a coleta de dados dos trabalhos incluídos na revisão, elaborou-se um instrumento baseado no proposto para revisão integrativa $^{(6)}$. A apresentação dos resultados e a discussão dos dados obtidos foram feitas de forma descritiva, por possibilitar a aplicabilidade da revisão elaborada e fornecer subsídios ao enfermeiro na tomada de decisão quanto ao melhor instrumento a se usar no trabalho com cuidadores de pacientes com AVE.

\section{RESULTADOS E DISCUSSÃO}

Concluída a busca nas bases de dados, selecionaram-se 23 trabalhos, nos quais foram identificadas 24 diferentes escalas para mensurar a sobrecarga de cuidadores de pacientes com AVE, como mostra o Quadro 2. Como mais citadas, incluíram-se o Caregiver Strain Index (CSI) ${ }^{(7-17)}$, a Caregiver Burden Scale $(\mathrm{CBS})^{(7-8,18-20)}$, o Caregiver Reaction Assessment $(\mathrm{CRA})^{(7,10,15,21)}$, o Sense of Competence Questionnaire (SCQ) ${ }^{(7-8,15)}$, o Relatives Stress Scale (RSS) $)^{(7-8,22)}$ e o Zarit Burden Interview $(\mathrm{ZBI})^{(8,23-24)}$.

Escalas para mensurar a sobrecarga de cuidadores de pacientes com AVE

1. Caregiver Strain Index (CSI) ${ }^{(7-17)}$

2. Caregiver Burden scale (CBS) $)^{(7,8,18,19,20)}$

3. Caregiver Reaction Assessment (CRA) $(7,10,15,21)$

4. Sense of Competence Questionnaire (SCQ) $)^{(7,8,15)}$

5. Relatives Stress Scale (RSS)

6. Zarit Burden Interview (ZBI) ${ }^{(8,24,25)}$

7. Bakas Caregiving Outcome Scale (BCOS) ${ }^{(7)}$

8. Braithwaite Burden Scale ${ }^{(7)}$

9. Burden Index: Personal Strain Index e Role Strain Index ${ }^{(29)}$

10. Burden Interview/Index (BI) ${ }^{(7)}$

11. Burnout Measurement ${ }^{(7)}$

12. Caregiver Burden Inventory (CBI) ${ }^{(12)}$

13. Caregiver Strain Scale (CSS) (7)

14. Caregiving Appraisal Scale (CAS) ${ }^{(30)}$

15. Caregiving Burden Scale $(\mathrm{CB})^{(7)}$

16. Carer Assessment $\mathrm{Scal}^{(7)}$

17. Cost of Care Index (CCl) ${ }^{(31)}$

18. Objective Burden Scale (OBS) ${ }^{(28)}$

19. Philadelphia Geriatric Center Caregiving Appraisal Scale ${ }^{(7)}$

20. Physical Caregiving Responsibility Inventory (PCRI) ${ }^{(7)}$

21. Questionnaire on Resources and Stress (QRS) ${ }^{(7)}$

22. Self-Rated Burden (SRB) ${ }^{(21)}$

23. Strain Scale ${ }^{(7)}$

24. Subjective Burden Scale (SBS) ${ }^{(28)}$

Quadro 2 - Escalas para mensurar a sobrecarga de cuidadores de pacientes com AVE.
Em relação ao tipo de revista na qual foram publicados os artigos incluídos, apenas quatro saíram em revista de enfermagem geral enquanto os demais trabalhos foram publicados em revistas de outras áreas. Isso evidencia que estudar a problemática dos cuidadores de pacientes com AVE tem sido notório por parte de outros profissionais da saúde e que o enfermeiro ainda não despertou para a urgência de publicar sobre essa temática.

Quanto ao tipo de delineamento de pesquisa dos trabalhos avaliados, com exceção de três pesquisas longitudinais ${ }^{(9,16,25)}$ e duas revisões ${ }^{(7-8)}$, tratava-se prioritariamente de estudos transversais (10 pesquisas), seguidos por seis ensaios clínicos randomizados. Tais estudos usavam a medida da sobrecarga do cuidador para avaliar a eficácia das intervenções propostas, desempenhadas em casa ou em clínicas de reabilitação, com processo de amostragem por conveniência e sem cálculo amostral. Os locais de realização predominantes foram países como Noruega (5), Reino Unido (4) e China (3).

Apesar de não ter sido identificada a melhor escala, nove trabalhos utilizaram e apontaram o Caregiver Strain Index como uma escala de fácil aplicação, por usar respostas dicotômicas (sim/não), conter um número pequeno de itens (13 assertivas), embora ainda não se tenha um ponto de corte para mensurar os valores da sobrecarga, sendo necessárias novas pesquisas com tais aspectos. Entretanto, alguns estudos ressaltaram que uma pontuação igual ou acima de sete pode indicar estar o cuidador sobrecarregado ${ }^{(9-10)}$.

Em determinado estudo no qual foi avaliada a reprodutibilidade da CSI, encontrou-se que o Coeficiente de Correlação Intraclasse (ICC) que avalia a confiabilidade interavaliadores esteve entre 0,84 a 0,97 e a média foi de $0,93^{(10)}$. Contudo, outra pesquisa apontou a necessidade de estudos longitudinais para mensurar a sobrecarga dos cuidadores de pacientes com AVE, pois dessa forma se poderia dizer com precisão quais aspectos estão interferindo mais ${ }^{(16)}$.

Os aspectos mais citados pelos cuidadores foram o confinamento, sentir-se oprimido, mudanças nos planos e desajustes familiares ${ }^{(11)}$. Já estudos realizados com cuidadores durante o período hospitalar mostraram que alguns pontos do CSI não são relevantes, como problemas financeiros e físicos ${ }^{(25)}$. Portanto, saber o valor da sobrecarga é importante para poder traçar intervenções ${ }^{(15)}$.

O Caregiver Burden Scale também foi uma escala amplamente adotada nas pesquisas. É composta por um questionário com 20 a 22 perguntas agrupadas em cinco dimensões (Tensão geral, Isolamento, Decepção, Envolvimento emocional e Ambiente). Estas dimensões abrangem áreas fundamentais para o cuidador, como saúde, bem-estar psicológico, relações pessoais, sobrecarga física, suporte social e ambiente. Para as respostas, utiliza-se a escala tipo Likert, com 3 a 5 itens. Não são especificados pontos de corte e o máximo de total da escala pode chegar a 60 ou 66 pontos. Entretanto, algumas limitações indicadas pelos estudos foram a necessidade de correlacionar essa escala com outros instrumentos para avaliar a sobrecarga e a dificuldade de compreensão tanto do grupo de cuidadores como dos avaliadores, motivado pelo uso de uma escala de Likert com quatro pontos ${ }^{(17)}$. Outros trabalhos 
apontaram a necessidade de se validar a escala para cada população por causa das diferenças culturais ${ }^{(19)}$.

Mais uma escala mencionada foi o Caregiver Reaction Assessment. Contém 24 itens e está dividida em cinco subescalas. Enquanto quatro subescalas avaliam os aspectos negativos do cuidar, como planos interrompidos, problemas financeiros, falta de apoio familiar e problemas de saúde, uma avalia possíveis aspectos positivos do cuidar, como a autoestima. Todas as pontuações da subescala são a média das contagens de item, variando de 1 a 5. Não há pontuação total. Utiliza-se a escala de Likert de cinco pontos, variando do discordo completamente até o concordo totalmente ${ }^{(14,21)}$.

Outro estudo encontrou que a reprodutibilidade das subescalas falta de apoio familiar e autoestima da CRA revelou-se insuficiente, pois a média do ICC foi de 0,67 e 0,58, respectivamente $^{(10)}$. Portanto, o uso de dois instrumentos é mais eficaz para mensurar a sobrecarga ${ }^{(20)}$.

Quanto ao Sense of Competence Questionnaire, abrange 27 itens divididos em três dimensões: satisfação com o receptor de cuidados, satisfação com a sua performance como cuidador e consequência do cuidar para a sua vida. Utiliza a escala de Likert de cinco pontos, variando do discordo completamente até o concordo totalmente. A pontuação total da escala pode variar de 27 a 108 pontos. Encontrou-se um alpha de Cronbach de 0,91 indicativo de alta consistência interna. Entretanto, a escala contém itens sensíveis que os cuidadores têm dificuldade para responder, logo pesquisas futuras são necessárias para adequá-los a cada cultura ${ }^{(21)}$.

A seguir, a Relatives Stress Scale, a qual avalia as atividades domésticas mais cansativas, os sentimentos negativos e angústia pessoal em relação ao paciente. Escores mais altos indicam maior estresse.

Enfim, a Zarit Burden Interview. Esta escala compreende 22 itens para mensurar a sobrecarga. Altos escores sugerem alta sobrecarga. Utiliza escala de Likert de cinco pontos, variável de nunca a sempre. Avalia os aspectos físico, psicológico, emocional, social e financeiro. No estudo para cuidadores de pacientes com AVE encontrou-se um ponto de corte de 25 que se mostrou acurado, ou seja, sensibilidade de $77 \%$ e especificidade de $72 \%$. Reconhecer o ponto de corte para o nível da sobrecarga permite estabelecer quando começar a implementar as intervenções. Uma vez que, alto valor de sobrecarga aumenta o risco para depressão ${ }^{(23)}$. Outros estudos mostraram apenas o nível de sobrecarga de uma área restrita do oeste do Japão, sendo necessário aplicar a escala para outras populações ${ }^{(24)}$.

$\mathrm{Na}$ presente revisão, 24 escalas foram identificadas para mensurar a sobrecarga do cuidador de pacientes com AVE. Destas, a CSI é a mais comum. Tal como nos outros trabalhos, foram mensurados aspectos de competência, sentimentos negativos, saúde física e mental, relação social, problemas sociais e aspectos econômico, sendo mais comuns escalas para avaliar a subjetividade da sobrecarga.

Em estudo sobre comparação entre as escalas CSI, CRA e SCQ, identificou-se que o uso de três escalas com itens parecidos confundia os cuidadores. Portanto, indicou-se o uso da CSI para estudos transversais, pois os itens eram melhores compreendidos pelos cuidadores. Não obstante, reforçaram ser indispensável pesquisa de validação de escala ${ }^{(14)}$.

Outro estudo ao avaliar a reprodutibilidade da CSI e da CRA mostrou que a reprodutibilidade foi boa para CSI e moderada para o CRA. A resolutividade foi moderada para o CSI e insatisfatória para o CRA. Uma indicação foi utilizar o CRA sem as escalas de apoio familiar e autoestima, pois elas confundem as respostas dos cuidadores, por serem muito pessoais ${ }^{(10)}$. Um ponto também limitador para o uso da CSI é não saber dos valores da sobrecarga antes do evento do $\mathrm{AVE}^{(12)}$.

Todavia, ao utilizar a Objective Burden Scale (OBS) e a Subjective Burden Scale (SBS) observou-se novamente a associação entre a sobrecarga e a depressão. Entretanto, o estudo limitou-se, pois foram avaliados pacientes com bom nível funcional; pelo fato de estudos transversais não fornecer uma boa compreensão de quais aspectos estão envolvidos na sobrecarga; e por se restringir à população coreana ${ }^{(26)}$.

Nos estudos de revisão encontrados, o CSI, o BI e a RSS foram as mais comuns para mensurar a sobrecarga. Todas as escalas mensuravam aspectos de competência, sentimentos negativos, saúde física e mental, relação social, problemas sociais e aspectos econômicos, mas não ficou clara a diferença de foco das escalas, pois é limitado o conhecimento sobre as propriedades psicométricas ${ }^{(7)}$.

Na Bakas Caregiving Outcome Scale (BCOS), o CBS e o CSI enfatizavam as consequências do cuidado. No Burden Interview/Index (BI), RSS, SCQ e CRA avaliavam o aspecto negativo da sobrecarga, enquanto SCQ, BCOS, Caregiving Burden Scale (CB) e CRA eram instrumentos multidimensionais para avaliar a sobrecarga. O BI e o CSI eram escalas unidimensionais ${ }^{(7)}$.

Ressalta-se que estudos longitudinais são importantes para avaliar a sobrecarga dos cuidadores, pois na grande maioria dos estudos a sobrecarga é vista apenas como um aspecto negativo ${ }^{(8)}$.

\section{CONCLUSÃO}

A utilização de escalas para mensurar a sobrecarga de cuidadores de pacientes com AVE é uma ferramenta importante para avaliar a eficácia de intervenções propostas para reduzir a tensão gerada ao desempenhar esse papel. Entretanto, ainda é escasso o conhecimento sobre as propriedades psicométricas das escalas. Portanto, torna-se essencial pesquisa de validação de escalas para nossa população, uma vez que as diferenças culturais existentes modificam os aspectos passíveis de interferir na sobrecarga dos cuidadores.

Como mencionado, a escala mais utilizada foi o Caregiver Strain Index, que é um instrumento já validado em diversos países e com alta consistência interna. Outras escalas bastante utilizadas foram o Caregiver Burden Scale, o Caregiver Reaction Assessment, o Sense of Competence Questionnaire, o Relatives Stress Scale e o Zarit Burden Interview.

Apesar da discussão sobre essa temática, a avaliação da sobrecarga é mais fidedigna quando se associa mais de um instrumento, mediante estudos longitudinais, pois essa medida não pode ser vista apenas como um aspecto negativo na vida dos cuidadores. 
O estudo limitou-se, sobretudo, pelo fato dos artigos avaliados nessa revisão não expor as recomendações ou dificuldades para o uso das escalas e por não trazer os aspectos psicométricos. Sugerem-se, pois, mais pesquisas para se identificar qual escala melhor se adapta aos cuidadores de pacientes com AVE e que esteja validada para o Brasil.

\section{REFERÊNCIAS}

1. Hare R, Rogers $H$, Lester $H$, Mcmanus RJ, Mant J. What do stroke patients and their carers want from community services? Fam Pract 2006;23:131-6.

2. Gomes WD, Resck ZMR. A percepção dos cuidadores domiciliares no cuidado a clientes com sequelas neurológicas. Rev Enferm UERJ 2009;17(4):496-501.

3. Pedreira LC, Lopes RLM. Cuidados domiciliares ao idoso que sofreu Acidente Vascular Cerebral. Rev Bras Enferm 2010;63(5):837-40.

4. Nietsche EA. Tecnologia emancipatória: possibilidade ou impossibilidade para a práxis de enfermagem? Florianópolis: UNJUí; 2000.

5. Whittemore $\mathrm{R}, \mathrm{Knafl} \mathrm{K}$. The integrative review: updated methodology. J Adv Nurs 2005;52(5):546-53.

6. Ursi ES. Prevenção de lesões de pele no perioperatório: revisão integrative da literatura. São Paulo. Dissertação [Mestrado em Enfermagem]- Escola de Enfermagem de Ribeirão Preto, Universidade de São Paulo; 2005.

7. Visser-Meily JMA, Post MWM, Riphagen II, Lindeman E. Measures used to assess burden among caregivers of stroke patients: a review. Clin Rehabil 2004;18(6):601-23.

8. Greenwood N, Mackenzie A, Cloud GC, Wilson N. Informal carers of stroke survivors - factors influencing carers: A systematic review of quantitative studies. Disabil Rehabil 2008;30(18):1329-49.

9. Visser-Meily A, Post M, Van de Port I, Maas C, ForstbergWarleby G, Lindeman E. Psychosocial functioning of spouses of patients with stroke from initial inpatient rehabilitation to 3 years poststroke: course and relations with coping strategies. Stroke 2009;40:1399-404.

10. Post M, Festen H, Van de Port I, Visser-Meily J. Reproducibility of the caregiver strain index and the caregiver reaction assessment in partners of stroke patients living in the Dutch community. Clin Rehabil 2007;21(11):1050-5.

11. Ilse IB, Feys H, Wit L, Putman K, Weerdt W. Stroke caregivers' strain: prevalence and determinants in the first six months after stroke. Disabil Rehabil 2008;30(7):523-30.

12. Tooth L, Mckenna K, Barnett A, Prescott C, Murphy S. Caregiver burden, time spent caring and health status in the first 12 months following stroke. Brain Inj 2005;19(12):963-74.

13. Blake $H$, Wyller TB, Thommessen B, Sødring KM, Sveen $U$, Pettersen AM, et al. Emotional well-being of close relatives to stroke survivors. Clin Rehabil 2003;17(4):410-7.

14. Exel NJA, Reimer WJMS, Reimer WBF, van den Berg B, Koopmanschap MA, van den Bos GAM. Instruments for assessing the burden of informal caregiving for stroke patients in clinical practice: a comparison of CSI, CRA, SCQ and self-rated burden. Clin Rehabil 2004;18(2):203-14.

15. Heuvel ETP, Witte LP, Schure LM, Sanderman R,
Meyboom-de Jong B. Risk factors for burn-out in caregivers of stroke patients, and possibilities for intervention. Clin Rehabil 2001;15(6):669-77.

16. Draper $\mathrm{P}$, Brocklehurst $\mathrm{H}$. The impact of stroke on the well-being of the patient's spouse: an exploratory study. J Clin Nurs 2007; 16(2):264-71.

17. Björkdahl A, Nilsson AL, Sunnerhagen KS. Can rehabilitation in the home setting reduce the burden of care for the next-of-kin of stroke victims? J Rehabil Med 2007;39(1):27-32.

18. Kao HF. Institutionalization in Taiwan: the role of caregiver gender. J Gerontol Nurs 2003;29(10):12-21.

19. Kao HF, Acton GJ. Conceptualization and psychometric properties of the caregiver burden scale in Taiwan. Issues Ment Health Nurs 2006;27(8):853-66.

20. Van Exel NJA, Brouwer WBF, van den Berg B, Koopmanschap MA, van den Bos GAM. What really matters: an inquiry into the relative importance of dimensions of informal caregiver burden. Clin Rehabil 2004;18(6):683-93.

21. Mackenzie A, Perry L, Lockhart E, Cottee E, Cloud G, Mann H. Family carers of stroke survivors: needs, knowledge, satisfaction and competence in caring. Disabil Rehabil 2007;29(2):111-21.

22. Draper B, Bowring G, Thompson C, van Heyst J, Conroy $\mathrm{P}$, Thompson J. Stress in caregivers of aphasic stroke patients: a randomized controlled trial. Clin Rehabil 2007;21(2):122-30.

23. Schreiner AS, Morimoto T, Arai Y, Zarit S. Assessing family caregiver's mental health using a statistically derived cut-off score for the Zarit Burden Interview. Aging Ment Health 2006;10(2):107-11.

24. Morimoto T, Schreiner AS, Asano H. Caregiver burden and health-related quality of life among Japanese stroke caregivers. Age Ageing 2003;32(2):218-23.

25. Blake $\mathrm{H}$, Lincoln NB, Clarke DD. Caregiver strain in spouses of stroke patients. Clin Rehabil 2003;17(3):312-7.

26. Suh M, Kim K, Kim I, Cho N, Choi H, Noh S. Caregiver's burden,depression and support as predictors of poststroke depression: a cross-sectional survey. Int J Nurs Stud 2005;42(6):611-8.

27. Teng J, Mayo NE, Latimer E, Hanley J, Wood-Dauphinee $S$, Côté $R$, et al. Costs and caregiver consequences of early supported discharge for stroke patients. Stroke 2003;34(2):528-36.

28. Lee JH, Friedmann E, Picot SJ, Thomas SA, Kim CJ. Korean version of the revised caregiving appraisal scale: a translation and validation study. J Adv Nurs 2007;59(4):407-15.

29. Mak AKM, Mackenzie A, Lui MHL. Changing needs of Chinese family caregivers of stroke survivors. J Clin Nurs 2007;16(5): 971-9. 